\title{
A Molecular Mechanism for mRNA Trafficking in Neuronal Dendrites
}

\author{
Jianguo Shan, ${ }^{1}$ Trent P. Munro, ${ }^{1}$ Elisa Barbarese, ${ }^{2}$ John H. Carson, ${ }^{3}$ and Ross Smith ${ }^{1}$ \\ ${ }^{1}$ Department of Biochemistry and Molecular Biology, University of Queensland, Queensland 4072, Australia, and Departments of ${ }^{2}$ Neuroscience and \\ ${ }^{3}$ Biochemistry, University of Connecticut Health Center, Farmington, Connecticut 06030
}

\begin{abstract}
Specific neuronal mRNAs are localized in dendrites, often concentrated in dendritic spines and spine synapses, where they are translated. The molecular mechanism of localization is mostly unknown. Here we have explored the roles of A2 response element (A2RE), a cis-acting signal for oligodendrocyte RNA trafficking, and its cognate trans-acting factor, heterogeneous nuclear ribonucleoprotein (hnRNP) A2, in neurons. Fluorescently labeled chimeric RNAs containing A2RE were microinjected into hippocampal neurons, and RNA transport followed using confocal laser scanning microscopy. These RNA molecules, but not RNA lacking the A2RE sequence, were transported in granules to the distal neurites. hnRNP A2 protein was implicated as the cognate trans-acting factor: it was colocalized with RNA in cytoplasmic granules, and RNA trafficking in neurites was compromised by A2RE mutations that abrogate hnRNP A2 binding. Coinjection of antibodies to hnRNP A2 halved the number of trafficking cells, and treatment of neurons with antisense oligonucleotides also disrupted A2RE-RNA transport. Colchicine inhibited trafficking, whereas cells treated with cytochalasin were unaffected, implicating involvement of microtubules rather than microfilaments. A2RE-like sequences are found in a subset of dendritically localized mRNAs, which, together with these results, suggests that a molecular mechanism based on this cis-acting sequence may contribute to dendritic RNA localization.
\end{abstract}

Key words: RNA trafficking; RNA localization; neuronal dendrites; hippocampal neurons; microinjection; confocal microscopy

\section{Introduction}

The development of cellular and organismal asymmetry requires selective localization of morphogenic proteins within cells. In oocytes, localization is often achieved through targeting of their mRNAs (Lasko, 1999; Mowry and Cote, 1999; Lipshitz and Smibert, 2000). RNA targeting has also been observed in mammalian somatic cells including glia, neurons, fibroblasts, and epithelia (Carson et al., 1998; Oleynikov and Singer, 1998; Bassell et al., 1999).

Two pathways for mRNA trafficking in somatic cells have been described in detail. In oligodendrocytes, RNAs containing a 21 nucleotide (nt) cis-acting sequence termed the heterogeneous nuclear ribonucleoprotein (hnRNP) A2 response element (A2RE), which was originally identified in the myelin basic protein mRNA (Ainger et al., 1997), or an 11 nt 5' segment of A2RE (A2RE11), are recruited to specific transport granules through association with hnRNP A2 (Ainger et al., 1997; Carson et al., 1998; Hoek et al., 1998; Munro et al., 1999). Kinesin- and microtubule-dependent granule movement from the soma into

\footnotetext{
Received June 25, 2003; revised Aug. 1, 2003; accepted Aug. 6, 2003.

This research was supported by an Australian National Health and Medical Research Council grant (R.S.) and by National Institutes of Health Grants NS19943 (E.B.) and NS15190 (J.H.C). This work was made possible by an equipment grant from the Wellcome Trust. We thank W. F. C. Rigby for the mouse anti-hnRNP A2 and M. Maggipinto and $Y$. Gao for technical assistance.

Correspondence should be addressed to Prof. Ross Smith, Department of Biochemistry and Molecular Biology, University of Queensland, Queensland 4072, Australia. E-mail: ross.s@uq.edu.au.

T. P. Munro's present address: Wellcome/Cancer Research UK Institute, Tennis Court Road, Cambridge CB2 1QR UK.

Copyright $\odot 2003$ Society for Neuroscience $\quad$ 0270-6474/03/238859-08\$15.00/0
}

the cell processes results in myelin basic protein mRNA translation at the cell periphery (Carson et al., 1997; Brumwell et al., 2002). A microfilament-dependent pathway for trafficking of $\beta$-actin mRNA in fibroblasts with different cis- and trans-acting factors has been described (Sundell and Singer, 1991; Farina et al., 2003; Oleynikov and Singer, 2003).

In neurons, a subset of mRNAs is localized to the dendrites (Landry et al., 1994; Steward, 1997; Kuhl and Skehel, 1998; Mohr, 1999; Kiebler and DesGroseillers, 2000; Gardiol et al., 2001; Kindler and Monshausen, 2002). Localized RNAs are often found colocalized with ribosomes and other translation apparatus components near dendritic spines (Steward and Levy, 1982; Chicurel et al., 1993; Knowles et al., 1996; Tiedge and Brosius, 1996; Torre and Steward, 1996). Some of these localized RNAs, such as that encoding the calcium-calmodulin-dependent protein kinase II $\alpha$ subunit, may participate in activity-related protein synthesis (Crino and Eberwine, 1996; Tongiorgi et al., 1997; Schuman, 1999; Steward and Worley, 2001), providing a mechanism for long-term potentiation (Frey and Morris, 1997; Steward and Halpain, 1999).

For most neuronally localized RNAs, the cis-acting targeting sequences are not well defined, often being associated with RNA segments that are hundreds of nucleotides in length. A few have been located within shorter RNA segments. The $\beta$-actin mRNA trafficking element falls within a $54 \mathrm{nt} 3^{\prime}$-untranslated region (UTR) region (Zhang et al., 2001; Turuchinapalli et al., 2003), and $62 \mathrm{nt}$ of the $5^{\prime}$ end is required for localization of the noncoding neuronal BC1 RNA (Muslimov et al., 1997). Embryonic lethal abnormal vision-like RNA binding proteins bind short AU-rich 
elements in many localized transcripts and may regulate their localization, stability, and translation (Kindler and Monshausen, 2002). Translin (testis-brain RNA-binding protein) binds the short $\mathrm{Y}$ and $\mathrm{H}$ sequences that are found in many neuronal transcripts (Severt et al., 1999). Finally, a 28 nt 3'-UTR cis-acting element has been proposed to mediate the dendritic localization of the mRNA encoding the $\alpha$-subunit of $\mathrm{Ca}^{2+} /$ calmodulindependent protein kinase II (CaMKII) (Mori et al., 2000), although other segments have also been suggested to possess this function (Blichenberg et al., 2001).

Few neuronal trans-acting factors have been identified. Here we show that microinjected RNA containing the short A2RE element is recruited to granules that are transported along the neurites of hippocampal neurons. This RNA targeting appears to be dependent on the trans-acting factor hnRNP A2: coinjection of hnRNP A2 antibody or treatment of neurons with antisense oligonucleotides diminishes RNA targeting in dendrites, and mutations in A2RE that weaken its interaction with hnRNP A2 interfere with transport. Disruption of the cytoskeleton with colchicine is shown to eliminate RNA transport, which is thus dependent on the maintenance of the microtubule network. Because several dendritically localized RNAs contain A2RE-like sequences (Ainger et al., 1997), interaction of this motif with hnRNP A2 may afford a general pathway for targeting these molecules.

\section{Materials and Methods}

Cell culture. Hippocampus dissected from embryonic day 18 Wistar rats was digested for $15 \mathrm{~min}$ at $37^{\circ} \mathrm{C}$ with $0.25 \%(\mathrm{w} / \mathrm{v})$ trypsin in HBSS, washed twice in HBSS, placed in DMEM supplemented with $10 \%(\mathrm{v} / \mathrm{v})$ fetal calf serum, and mechanically dissociated by trituration (Goslin and Banker, 1998). The cells were plated at low density $\left(600\right.$ cells $\left./ \mathrm{mm}^{2}\right)$ on poly-L-lysine-coated (Sigma, St. Louis) glass-bottomed microwells (MatTek, Ashland, MA). After $2 \mathrm{hr}$, when neurons had attached to the substrate, $2 \mathrm{ml}$ of medium containing Neurobasal, N2 supplements (1:100 dilution), B27 supplements (1:50 dilution), and 5\% (v/v) Gentamicin was added, and the cells were incubated at $37^{\circ} \mathrm{C}$ in $95 \%$ air $/ 5 \% \mathrm{CO}_{2}$. The medium was replaced every other day. All reagents were from Life Technologies (Rockville, MD), except where noted otherwise. The cells were identified as neurons by microtubule-associated protein (MAP) $2 \mathrm{immu}-$ nostaining and their morphology, and excluded as oligodendrocytes or astrocytes by their lack of immunofluorescence with antibodies to myelin basic protein and glial cell fibrillary acidic protein, respectively.

In vitro transcription. Vectors, derived from the plasmid pNKT7, containing A2RE (5'GCCAAGGAGCCAGAGAGCAUG3'), A2RE11 (5'GCCAAGGAGCC3'), A8G (5'GCCAAGGGGCC3'), G9A (5'GCCAAGGAACC $\left.3^{\prime}\right)$ or MAP 2A (5'GCCAAGGAGUCAGAAGACAUG3') sequences inserted between the green fluorescent protein (GFP) open reading frame and the segment encoding the poly(A) tail were constructed in our laboratory (Munro et al., 1999). The purified plasmids were linearized with BsaW1 (New England Biolabs, Beverly, MA) and transcribed in vitro in the presence of Alexa Fluor 488-5'-UTP (Molecular Probes, Eugene, OR) with an Ampliscribe kit (Epicenter Technologies, Madison, WI). The resultant RNAs were precipitated with $7.5 \mathrm{M}$ ammonium acetate. The precipitate was washed twice with $70 \%$ ethanol and then dissolved in diethyl pyrocarbonate (DEPC)-treated water. The RNAs were further purified on polyacrylamide gel matrix columns (Micro Bio-Spin Columns P-30 Tris, RNase-free; Bio-Rad, Cambridge, MA), and an aliquot of each was subjected to electrophoresis on an agaroseformaldehyde gel to verify purity and estimate the RNA concentration. In real-time experiments, the RNA $(1.0 \mathrm{mg} / \mathrm{ml})$ was mixed with Texas Red-conjugated dextran (10 kDa; Molecular Probes) and filtered through an $0.2 \mu \mathrm{m}$ spin filter (Millipore, Bedford, MA) just before microinjection. In all other experiments, the RNA was diluted with DEPCtreated water to a final concentration at $0.5 \mathrm{mg} / \mathrm{ml}$ and then filtered through an $0.2 \mu \mathrm{m}$ filter before injection.
RNA microinjection and visualization. After 7-14 d in culture, differentiated hippocampal neurons were microinjected using Eppendorf (Westbury, NY) or Compic Inject (Cellbiology Trading, Hamburg, Germany) micromanipulators attached to Zeiss Axiophot inverted microscopes (Carl Zeiss, Oberkochen, Germany). Microinjection needles, with a tip of $\sim 0.5 \mu \mathrm{m}$, were produced in a Flaming Brown micropipette puller (Sutter Instruments, San Raphael, CA). The RNA solution was between 0.5 and $1.0 \mathrm{gm} / \mathrm{l}$. Increasing the concentration of the RNA injected into live 7-d-old hippocampal neurons from 0.15 to $1.0 \mathrm{mg} / \mathrm{ml}$ did not change the distribution of the fluorescently labeled RNA. The injection pressure was $40 \mathrm{hPa}$, and the holding or back-pressure was $20 \mathrm{hPa}$. The injection period was restricted to $1 \mathrm{hr}$ per microplate. After injection, the cells were incubated at $37^{\circ} \mathrm{C}$ for $30 \mathrm{~min}$ to allow peripheral transport to occur. Cells were then imaged directly, or after fixing and immunolabeling, on Zeiss LSM 410 or 510, or Bio-Rad MRC 800, laser scanning confocal microscopes equipped with $63 \times$ [1.4 numerical aperture (NA)] and $60 \times(1.4$ $\mathrm{NA}$ ) lenses. The parameters on the microscopes were adjusted to minimize bleed-through using small beads with green fluorescent label and larger beads with red fluorescent label. All images were created by superimposition of z-series.

Image analysis. Digital images collected from the confocal microscopes were processed using Adobe Photoshop (Adobe Systems, Mountain View, CA). The identification of images as demonstrating that there was, or was not, transport was assessed independently by two (sometimes three) observers who were blind to the nature of the injected RNA. The images were presented to the scorers in random order. In colocalization experiments, images were analyzed using Image-Pro Plus (Media Cybernetics, Silver Spring, MD). The granule size was defined as $3 \times 3$ pixels (corresponding to $0.5 \times 0.5 \mu \mathrm{m})$ (Knowles et al., 1996). A typical granule was selected manually, and the other granules in this channel were selected automatically by the software, which then measures the intensities in both channels (red and green) for these $3 \times 3$ pixel blocks. This process was reiterated several times starting with the initial manual selection in either channel, with parallel results.

Immunocytochemistry. Cells were washed with PBS and fixed for 20 min in $3.7 \%$ paraformaldehyde (Sigma) in PBS. After they were washed with PBS, the cells were permeabilized by incubation for $2 \mathrm{~min}$ in $0.1 \%$ Nonidet P-40 (Sigma) in PBS, washed, and then incubated in 5\% guinea pig serum (Life Technologies) in PBS for $10 \mathrm{~min}$. To visualize neurites, the cells were incubated for $30 \mathrm{~min}$ in mouse anti-MAP2 (Sigma), washed, and incubated for $30 \mathrm{~min}$ in Texas Red-conjugated goat antimouse IgG (Sigma). Finally, 70\% glycerol containing the antifading agent 1,4-diazabicyclo[2,2,2]octane was added to the cells before imaging.

Coinjection of antibodies to hnRNP A2. A2RE-GFP RNA was injected along with anti-hnRNP A2. The antibody solution $(3.2 \mathrm{mg} / \mathrm{ml})$ was mixed with an equal volume of the RNA $(1 \mathrm{mg} / \mathrm{ml})$ and water in the injection pipette. The murine monoclonal antibody to hnRNP A2 (EF67) was produced against, and is specific for, the C-terminal 17 amino acid residues of the protein (Nichols et al., 2000).

Antisense oligonucleotide treatment of neurons. Rat hippocampal neurons were incubated for $48 \mathrm{hr}$ in medium containing $8 \mu \mathrm{M}$ oligodeoxyribonucleotide. The sense and antisense oligonucleotides, 5'AAATCTGACCAGCATTTTTAT3' and 5'ATAAAAATGCTGGTCAGATTT3', respectively, were "Optimized Antisense Oligos" (Oligos Etc., Wilsonville, OR). hnRNP A2 levels in the treated neurons were examined by electroblotting, using tubulin as an internal standard for protein loading on the SDS-polyacrylamide gel, and by immunofluorescence microscopy. For electroblotting, treated or untreated neurons in microplates $\left(600\right.$ cells $\left./ \mathrm{mm}^{2}\right)$ were collected with protein extraction buffer $(20 \mathrm{~mm}$ HEPES, $0.65 \mathrm{M} \mathrm{KCl}, 2 \mathrm{~mm}$ EGTA, $1 \mathrm{~mm} \mathrm{MgCl}_{2}$, 2 м glycerol, $14.3 \mathrm{~mm}$ $\beta$-mercaptoethanol, 8.7 mм NP-40, 12.1 mm deoxycholic acid, 1 mм PMSF, and $21 \mu \mathrm{m}$ leupeptin, $0.5 \% \mathrm{SDS}, \mathrm{pH}$ 7.4). After centrifugation at $13,000 \times g$ for $15 \mathrm{~min}$ at $4^{\circ} \mathrm{C}$, the supernatant proteins were resolved by SDS-PAGE and then electroblotted onto Immobilon polyvinylidene difluoride membrane (Millipore, Sydney, Australia). The blots were developed with a mouse antibody to hnRNP A2 (as above; used at a 1:2000 dilution) or with rabbit antibody to $\alpha$-tubulin (used at 1:2000 dilution) as a control for protein loading, followed by horseradish peroxidase-conjugated secondary antibod- 


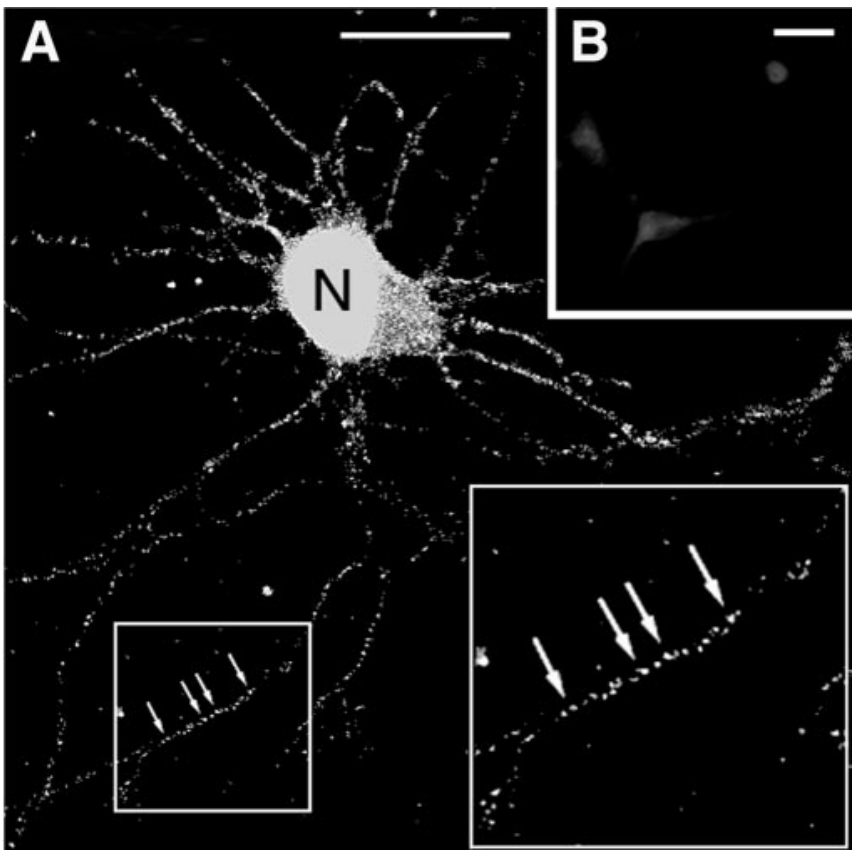

Figure 1. Location of hnRNP A2 in rat hippocampal neurons. A, Confocal microscope image of hnRNP A2 in the nuclei ( $\mathrm{N}$ ) and neurites (arrows) of fixed hippocampal neurons. The protein was detected using a mouse primary antibody and an FITC-labeled goat anti-mouse secondary antibody. The endogenous hnRNP A2 is visible in granules distributed throughout the neurites. The inset at right is a twofold magnification of the region boxed at left. Scale bar, $15 \mu \mathrm{m} . B, A$ control in which purified recombinant hnRNP A2 was added to the primary antibody before it was used for immunodetection of hnRNP A2 in neurons. Scale bar, $15 \mu \mathrm{m}$.

ies and enhanced chemiluminescence detection. For immunofluorescence microscopy, the neurons were fixed and treated with the hnRNP A2 antibody, followed by a fluorescein isothiocyanate-conjugated goat anti-mouse IgG.

Cytoskeleton disruption. To depolymerize microtubules, cells were treated with colchicine ( $10 \mu \mathrm{g} / \mathrm{ml}$; Sigma) in culture medium at $37^{\circ} \mathrm{C}$ for 30 min before microinjection with labeled A2RE RNA, fixation in paraformaldehyde, permeabilization, and immunolabeling. Cytochalasin (5 $\mu \mathrm{g} / \mathrm{ml}$; Sigma) was added to the cell medium for $2 \mathrm{hr}$ at $37^{\circ} \mathrm{C}$ to disrupt microfilaments. In each case, the medium in a control plate was replaced with normal culture medium after colchicine or cytochalasin treatment and then incubated at $37^{\circ} \mathrm{C}$ overnight before microinjection; RNA trafficking in these cells was indistinguishable from trafficking in untreated cells. The morphology and MAP2 distribution in neurons treated with either compound appeared to be unaffected.

\section{Results}

\section{hnRNP A2 is present in cytoplasmic granules in neurons}

Previous studies have established that hnRNP A2, which was originally identified as a component of the "core particles" associated with RNA packaging and processing in the nucleus (Dreyfuss et al., 1993), is a trans-acting factor for cytoplasmic trafficking of A2RE-containing RNA in oligodendrocytes (Hoek et al., 1998; Munro et al., 1999). In neurons, hnRNP A2 is located in the nucleus and in large granules distributed along the neurites (Fig. $1 A$ ). These granules are similar in size and number to those that have been implicated in intracellular transport in oligodendrocytes (Munro et al., 1999), suggesting that hnRNP A2 may also function as a neuronal trans-acting RNA trafficking factor. The specificity of the immunostaining was demonstrated by addition of purified, recombinant hnRNP A2 to the antibody solution used for immunostaining (Fig. $1 B$ ).
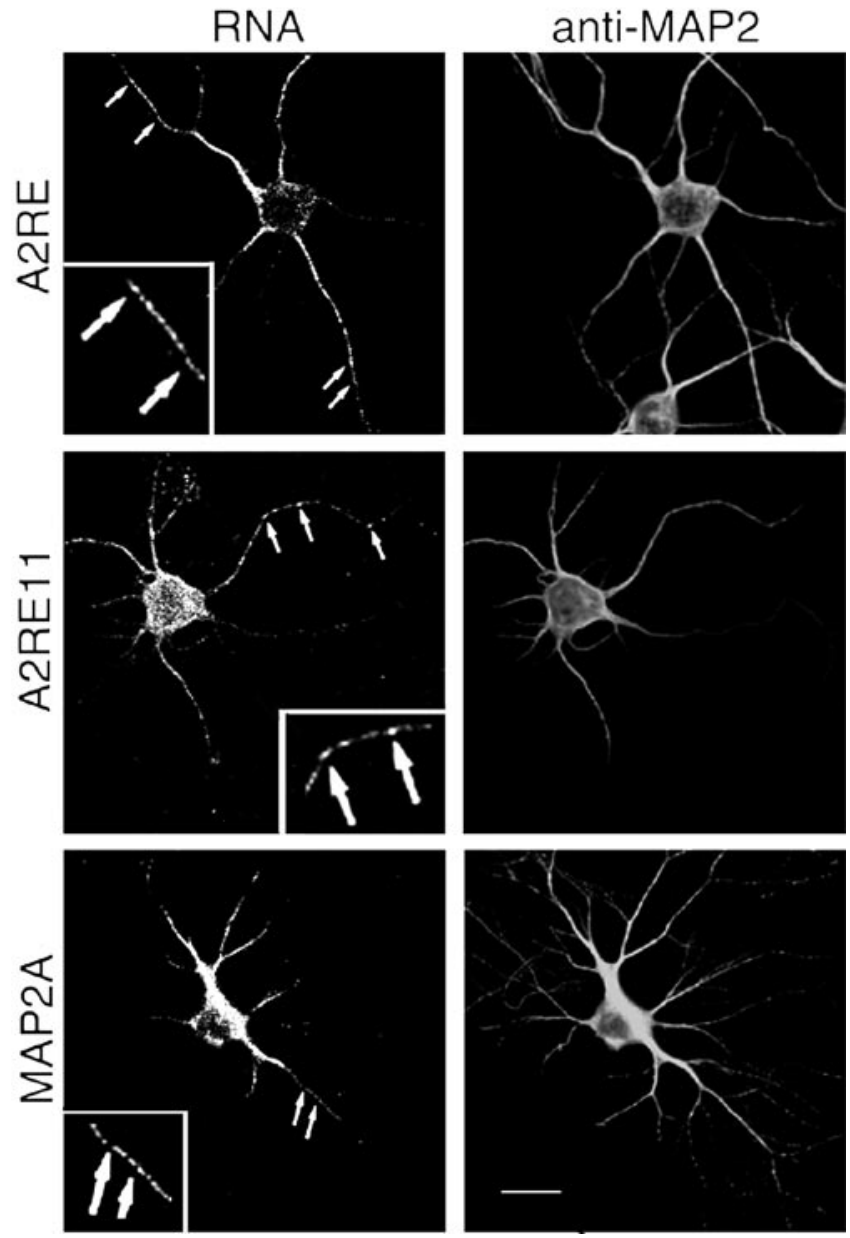

Figure 2. Transport of RNAs containing A2RE and A2RE-like elements in hippocampal neurons. Images of neurons in primary culture microinjected with fluorescently tagged RNA. After allowing 30 min after injection for RNA transport, the cells were fixed and stained for MAP2 protein to define the cell morphology. Dual-channel confocal microscopy was used to visualize the RNA (left panels) and protein (right panels). Each of the injected RNAs contained either A2RE or an A2RE-like element in the 3' UTR. RNAs bearing A2RE, A2RE11, or MAP2A elements were detected in granules in the soma and throughout the neurites (arrows). The insets show magnified regions. Scale bar, $15 \mu \mathrm{m}$.

\section{A2RE-containing RNA is transported in neurons}

Fluorescent RNA was microinjected into the soma of hippocampal neurons. The subcellular distribution of the microinjected RNA was subsequently analyzed in fixed cells (Figs. 2, 3) and in live cells (Fig. 4). RNA that lacked a localization sequence was retained in the soma 30-60 min after injection (data not shown). The same RNA containing the A2RE or the A2RE11 sequence immediately before the poly(A) tail was transported into the dendrites in discrete granules (Figs. 2, 4A). In Figure 4, the processes that are filled with Texas Red-conjugated dextran but exclude the injected green RNA may correspond to axons. The A2RE sequence used in these experiments is derived from myelin basic protein mRNA. Experiments were also performed with an A2RElike sequence derived from the neuronal MAP2A mRNA, with the same outcome (Fig. 2). The MAP2A A2RE sequence used in these experiments is located in the open reading frame (Ainger et al., 1997) and is sufficient for cytoplasmic trafficking. Thus, as in oligodendrocytes, the presence of the A2RE11 or A2RE-like sequences mediates RNA transport in these neurons (Figs. 3B, $4 B$ ). A separate dendritic localization element has been identified in the 3'UTR of MAP2 (Blichenberg et al., 1999), but there is no 

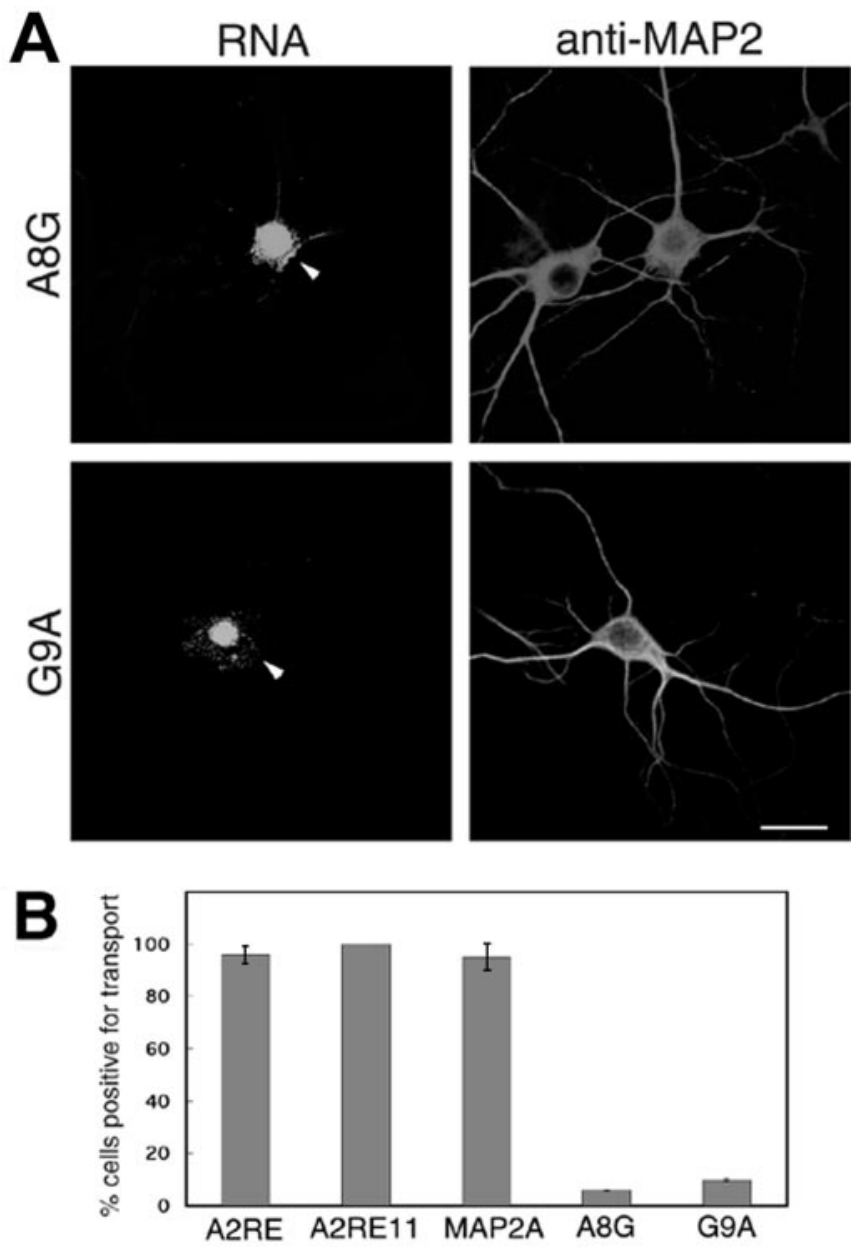

Figure 3. Mutations within the A2RE abolish trafficking in neurites. $A$, RNAs bearing the mutated elements A8G and G9A, which do not bind hnRNP A2, were observed only in the soma and proximal neurites (left panels, arrowheads). MAP2 was detected using mouse antibody and a tetramethylrhodamine isothiocyanate (TRITC)-labeled goat anti-mouse secondary antibody (right panels). Scale bar, $15 \mu \mathrm{m}$. B, Percentage of transport-positive cells for each of the exogenous RNAs in $A$ (this Figure) and Figure 2. For each RNA, at least 40 cells were imaged for analysis. The error bars represent the SDs derived from the binomial distribution.

obvious A2RE-like sequence within this segment. Dendritic localization of MAP2 mRNA may require both elements.

Introduction of mutant A2RE11 sequences containing either of two point mutations, A8G and G9A, known to interfere with binding to hnRNP A2 (Munro et al., 1999) resulted in retention of these RNAs in the cell body, suggesting that hnRNP A2 is involved in their trafficking. There are few RNA-containing granules in the neurites of these cells (Figs. 3A, 4A). The labeling evident in the nuclei results from uptake of small amounts of free fluorophore (Alexa-labeled UTP), which is difficult to remove from the in vitro transcribed RNA, and is not relevant to the current study.

\section{Antibodies to hnRNP A2 and antisense oligonucleotides interfere with trafficking}

Coinjection of A2RE-containing RNA with a monoclonal antibody to hnRNP A2 resulted in reduced trafficking (Fig. 5). In multiple experiments, RNA containing the $21 \mathrm{nt}$ A2RE, coinjected with water or antibodies to irrelevant proteins, was transported in $70 \%$ or more of the neurons, in accord with our other experiments on neurons (Fig. $4 B$ ). In contrast, $43 \%$ of 71 cells
A
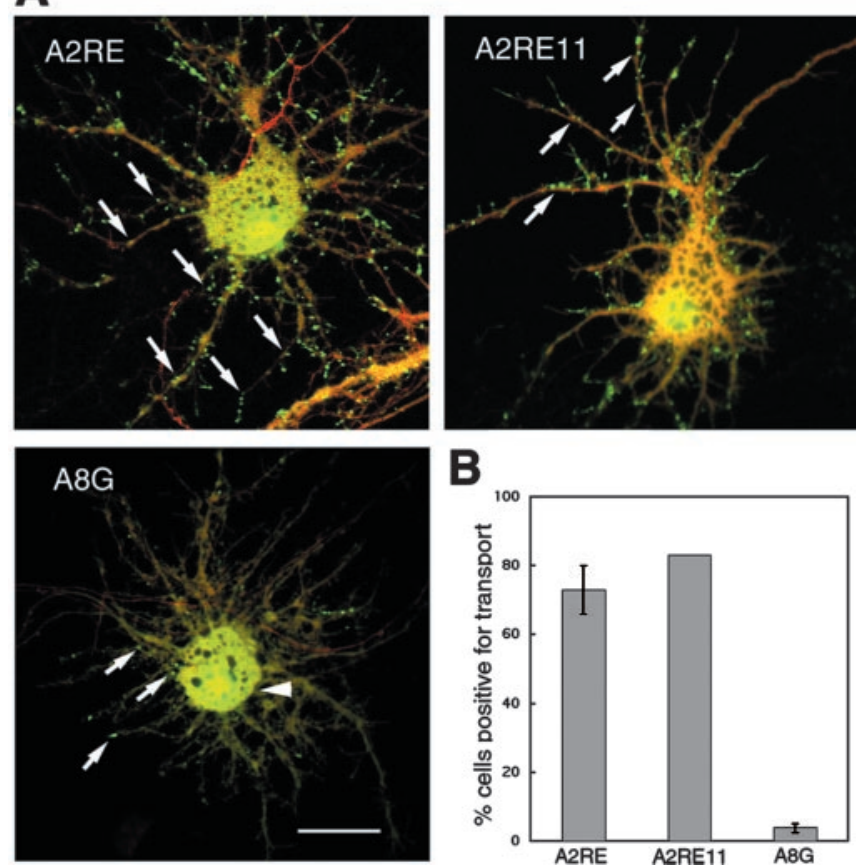

B

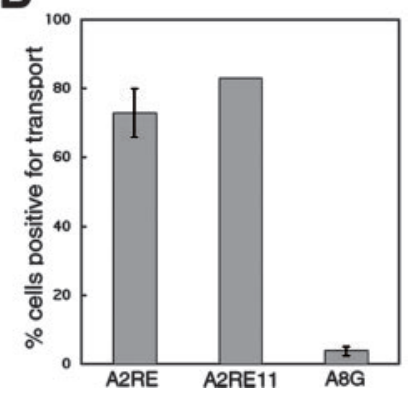

Figure 4. Transport of A2RE-containing RNAs visualized in live neurons. Hippocampal neurons were microinjected with fluorescently labeled RNA (green) and Texas Red-labeled $10 \mathrm{kDa}$ dextran (red), and after 30 min their distribution was determined by dual-channel confocal microscopy. A, Images of neurons microinjected with RNA. Exogenous RNAs containing A2RE or A2RE11 were observed to assemble into granules and move along the processes (arrows), whereas the A8G RNA did not assemble into granules but was distributed diffusely in the soma and proximal neurites (arrowhead) with only an occasional granule visible in the processes (arrows). Scale bar, $10 \mu \mathrm{m}$. B, Percentage of transport-positive cells for each of the microinjected RNAs in $A$. Error bars represent the SDs. For A2RE and A8G RNA, $>20$ cells were imaged for analysis. No error bar is given for A2RE11 because the number of cells imaged was small.

imaged after coinjection of RNA and antibody demonstrated RNA trafficking.

Neurons exposed to $8 \mu \mathrm{M}$ antisense oligonucleotide directed against the translational start site for hnRNP A2 showed $>30 \%$ reduction in expression on Western blots (Fig. 6A), with a clear reduction in the protein fluorescence in the dendrites (Fig. $6 B$ ). The cell and process densities in the two images in Figure $6 B$ were similar, as assessed by MAP2 immunofluorescence. Antisense treatment, but not sense treatment, affected the trafficking of RNA in the hippocampal neurons. RNAs containing A2RE, A2RE11, or the MAP2A A2RE-like sequence were transported in sense-treated cells but to a lower extent in antisense-treated cells (Figs. 4D, 6C). Previous experiments with oligodendrocytes yielded qualitatively similar results, although hnRNP A2 expression in these cells showed greater suppression by treatment with antisense oligonucleotide (Kwon et al., 1999; Munro et al., 1999).

\section{hnRNP A2 colocalizes with A2RE-containing RNA}

If hnRNP A2 is a trans-acting factor for RNA trafficking, it may be colocalized with A2RE-containing transcripts in transport granules. The distribution of hnRNP A2 was examined using confocal microscopy in cells microinjected with RNA containing, or lacking, the transport signal. Exogenous RNA containing A2RE or A2RE11 was colocalized with hnRNP A2 in granules within the neurites (Fig. 7A), whereas RNA lacking A2RE was found exclusively in the soma (data not shown). Automated image analysis demonstrated a close correlation between levels of fluorescence from microinjected RNA and hnRNP A2, especially in the neu- 

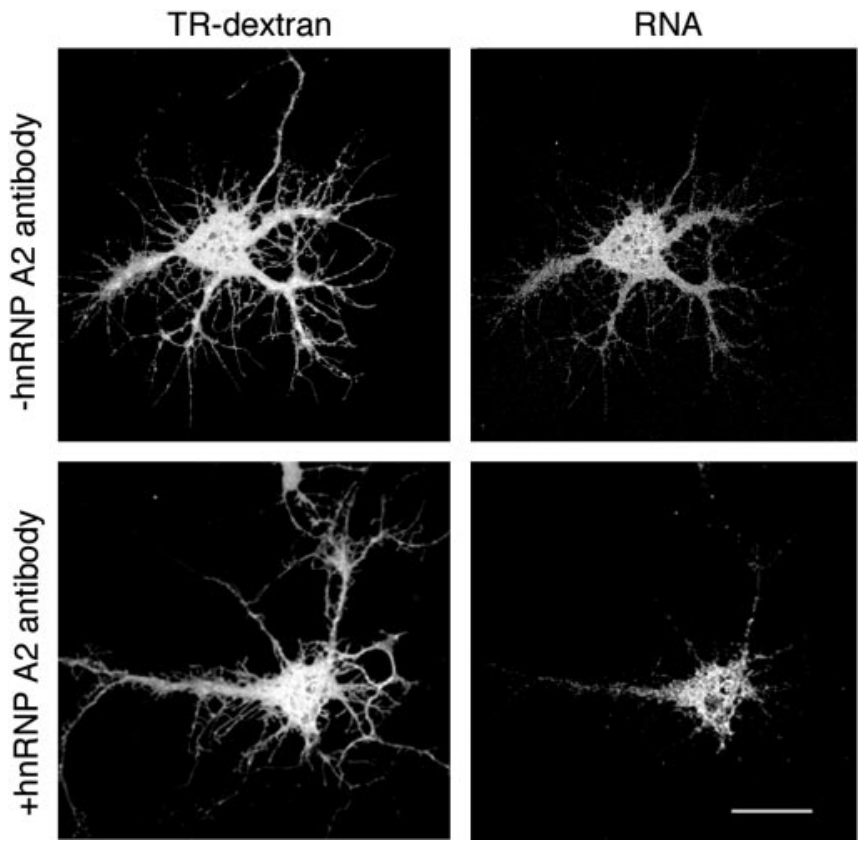

Figure 5. Inhibition of RNA trafficking by antibodies to hnRNPA2. A2RE-containing RNA was microinjected into cultured neurons either without (top) or with (bottom) antibody. The cell morphology is shown by the distribution of Texas Red-conjugated dextran (left). The RNA is labeled with Alexa Fluor-UTP (right) as described in Materials and Methods, and the images are from live cells. Scale bar, $15 \mu \mathrm{m}$.

rites (Fig. $7 B$ ), indicating that A2RE RNA and hnRNP A2 are present in the same proportions in different granules. RNA containing the MAP2A A2RE-like sequence showed a similar colocalization with hnRNP A2 (data not shown).

\section{Colchicine eliminates RNA trafficking}

Previous work showed that cellular exposure to the microtubuledisrupting agents nocodazole or colchicine inhibited RNA localization in oligodendrocytes (Carson et al., 1997). Hippocampal neurons treated with colchicine for $30 \mathrm{~min}$ did not transport microinjected A2RE-containing RNA and had decreased numbers of hnRNP A2 granules in the processes, yet the overall cell morphology appeared normal (Fig. 8); hnRNP A2-containing granules are evident (arrows, top right) but they do not contain microinjected A2RE RNA (arrows, top left). By contrast, disruption of microfilaments with cytochalasin had no effect on RNA trafficking or hnRNP A2 distribution. Thus A2RE-hnRNP A2 granule trafficking in neurons is dependent on the presence of intact microtubules but independent of microfilament organization. This contrasts with $\beta$-actin RNA localization in fibroblasts, which is dependent on microfilaments (Sundell and Singer, 1991) but parallels the trafficking of this mRNA in microtubuleassociated granules in neurons (Bassell et al., 1998).

\section{Discussion}

\section{An A2RE-hnRNP A2 pathway functions in neurons}

Our experiments characterize an RNA trafficking pathway in hippocampal neurons that is mediated by the A2RE cis-acting sequence and its cognate trans-acting trafficking factor, hnRNP A2. This pathway is microtubule dependent and parallels an RNA trafficking pathway described previously in oligodendrocytes (Carson et al., 1998; Hoek et al., 1998; Munro et al., 1999). Because A2RE-like sequences have been identified in various localized mRNAs, this may represent a general pathway for targeting
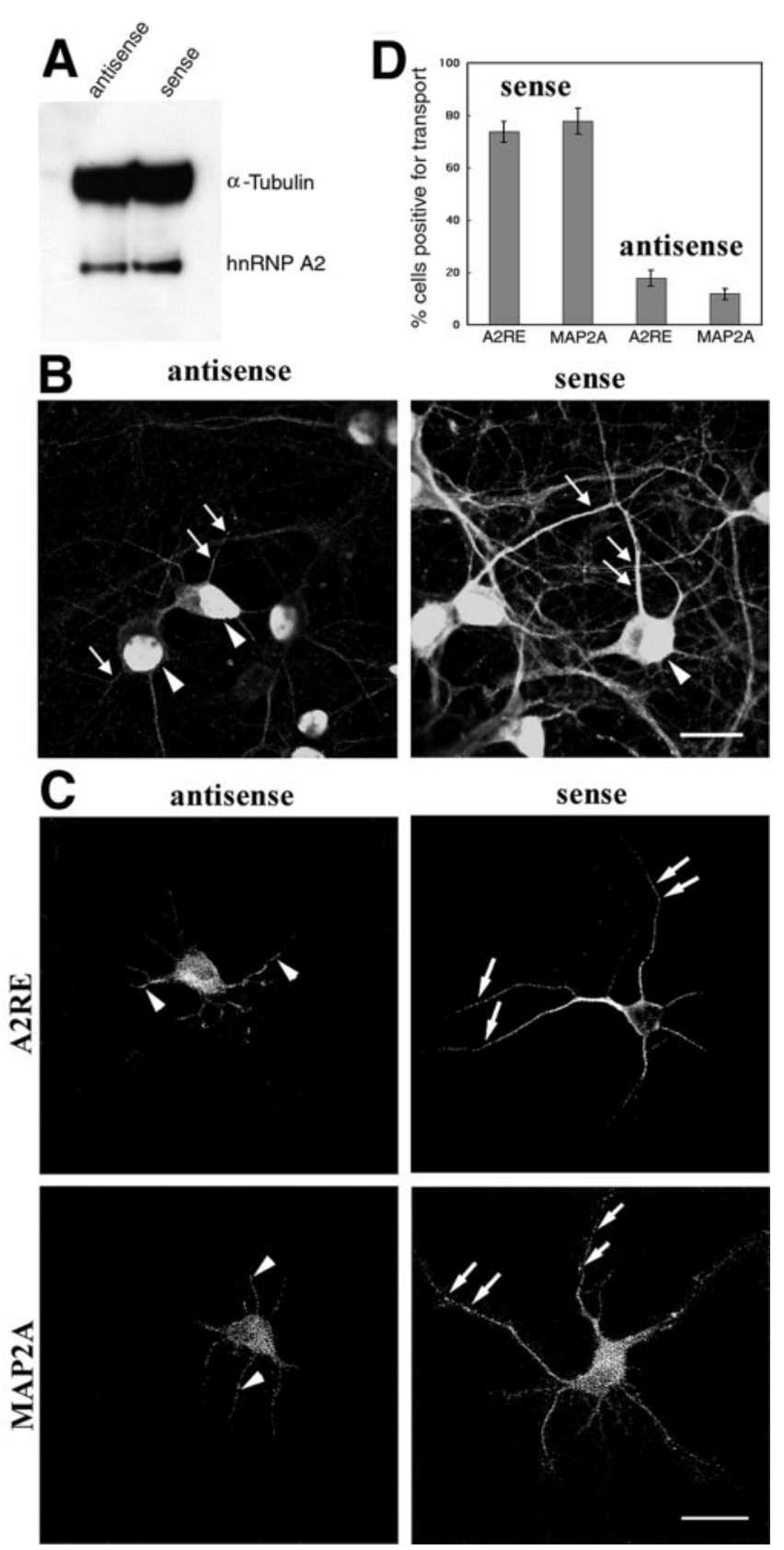

Figure 6. Effects of antisense oligonucleotide treatment on hnRNP A2 distribution and trafficking of A2RE-containing RNA. A, Western blot of hnRNP A2 in sense and antisense oligonucleotide-treated neurons with $\alpha$-tubulin used as control for protein loading on the gel. $B$, Intracellular distribution of hnRNP A2 in hippocampal neurons after hnRNP A2 sense and antisense treatment. In sense-treated cells, the protein is observed in the soma (arrowhead) and as granules in the processes (arrows). In antisense-treated cells, the hnRNP A2 is confined primarily to the nucleus (arrowheads), with markedly lower levels in the processes (arrows). The same confocal microscopy data collection and image processing parameters were used for both samples. In both images the maximal brightness in the nucleus has been set just below 255 , ensuring that all pixels are within the dynamic range of the imaging system, permitting direct comparison of the relative fluorescence intensities in the neurites in the two images. $C$, Representative images of hippocampal neurons microinjected with fluorescently labeled RNAs containing A2RE or the A2RE-like element of MAP2A after hnRNP A2 sense and antisense treatment. Injected RNAs were detected $50 \mu \mathrm{m}$ or more from the cell bodies in sense-treated cells (right panels, arrows). In antisense-treated cells, RNAs were observed only in the proximal processes (left panels, arrowheads). D, Percentage of transport-positive, sense- and antisensetreated cells injected with RNA containing A2RE or the cognate sequence from MAP2A RNA. Error bars represent the SDs. Scale bar, $15 \mu \mathrm{m}$. 
RNAs to the plus ends of microtubules in different cell types. Together with the recent report of formation of a complex between the $\beta$-actin mRNA zipcode and the zipcode binding protein (ZBP1) that is required for localization of this RNA to neuronal growth cones (Zhang et al., 2001), these studies present the first identification of proteins that bind to well defined cis-acting elements and are required for RNA localization in neurons.

\section{hnRNP A2 is abundant in the CNS and} is multifunctional

The hnRNP proteins were discovered as constituents of core particles in HeLa cells. The primary function of these nuclear particles is in packaging and processing of RNA; the hnRNPs are therefore concentrated in the nuclei of many cell types, particularly in a few tissues such as testis, lung, spleen, and brain. These proteins are not confined, however, to cell nuclei. In the brain they are evident in the processes of astrocytes, oligodendrocytes (Munro et al., 1999), and neurons (Fig. 1A), and there is accumulating evidence that they have distinct nuclear and extranuclear roles (Shyu and Wilkinson, 2000). In the cytoplasm of oligodendrocytes they are found in transport granules that also contain the molecular motors dynein and kinesin, ribosomes, and other molecules required for translation (Barbarese et al., 1995).

Mutational analysis and antisense oligonucleotide studies have implicated cytoplasmic hnRNP A2 (Munro et al., 1999) in RNA trafficking. Some mutations in the A2RE element markedly lowered its affinity for hnRNP A2. The two mutations that had the most pronounced effect in vitro were A8G and G9A. Oligonucleotides with these mutations were found not to bind human recombinant or rat brain hnRNP A2 in biosensor, pull-down, and electrophoretic mobility shift experiments, and these point mutations within a long RNA molecule sufficed to eliminate RNA transport in oligodendrocytes (Munro et al., 1999) and neurons (Figs. 3, 4). Antisense oligonucleotide treatment of neurons resulted in concentration of hnRNP A2 in the nucleus, leaving little in the neurites. By contrast, in oligodendrocytes, treatment with an equivalent oligonucleotide markedly reduced the levels of hnRNP A2 throughout these cells, perhaps reflecting a higher rate of export of this protein to the cytoplasm where it may be degraded and not replaced in the antisense-treated cells. In both cell types, however, antisense treatment compromised A2RE-dependent RNA trafficking. These antisense experiments were supported by others in which the population of cells transporting A2RE-containing RNA into the dendrites was diminished by injection of antibodies to hnRNP along with the RNA, presumably by reduction of the amount of hnRNP A2 available for binding to the RNA.

\section{Many localized RNAs, including dendritic RNAs, contain A2RE-like elements}

Numerous mRNAs are localized in dendrites. For example, dendritic localization of $\alpha$-CaMKII and its mRNA plays a pivotal role in synaptic plasticity, facilitating local control of mRNA stability and translation, and enzymatic activity, regulated by synaptic activity (Mayford et al., 1996; Mori et al., 2000; Rook et al., 2000; Aakalu et al., 2001; Lisman et al., 2002; Miller et al., 2002). Disruption of the $\alpha$-CaMKII dendritic localization element in the $3^{\prime} \mathrm{UTR}$ results in restriction of the mRNA to the soma of mouse neurons, a marked reduction of the protein in dendrites and particularly in postsynaptic densities, and reduction in late-phase long-term potentiation and memory consolidation (Miller et al., 2002). Other localized neuronal RNAs include noncoding RNAs (Tiedge et al., 1993; Muslimov et al., 1997), those encoding cytoskeletal proteins such as MAP2 (Bruckenstein et al., 1990), Arc (Steward et al., 1998), and $\beta$-actin (Bassell et al., 1998), enzymes including tyrosine receptor kinase B (Tongiorgi et al., 1997), neurotrophic factors (Tongiorgi et al., 1997), and neurotransmitter receptor subunits (Racca et al., 1997). As in oligodendrocytes, localization of these neuronal RNAs is generally mediated by microtubule-dependent movement of ribonucleoprotein granules (Wilhelm and Vale, 1993; Knowles et al., 1996; Bassell et al., 1998; Kohrmann et al., 1999). The A2RE-like segments of the mRNAs encoding the neuronal proteins MAP2A, Arc, and GABA receptor $\alpha$-subunit bind hnRNP A2 in vitro, and the MAP2A A2RE-like element, GCCAAGGAGUC (bases identical in A2RE underlined), supported RNA trafficking in oligodendrocytes processes (Munro et al., 1999). The MAP2A element also supported RNA dendritic localization in neurons (Fig. 2), and it is likely that the other A2RE-containing RNAs will also be localized by interaction with hnRNP A2.

Potential A2RE-like trafficking signals have been identified in other dendritically localized neuronal mRNA including those encoding rat $\alpha$-CaMKII, mouse neurogranin (GCCAAGGACCC), rat Arc (GCUGAGGAGGA), noncoding rat RNA BC1 (GL-

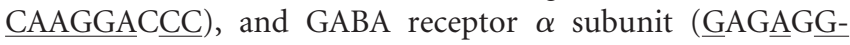
GAGCC). The 3'UTR of rat $\alpha$-CaMKII mRNA contains the 

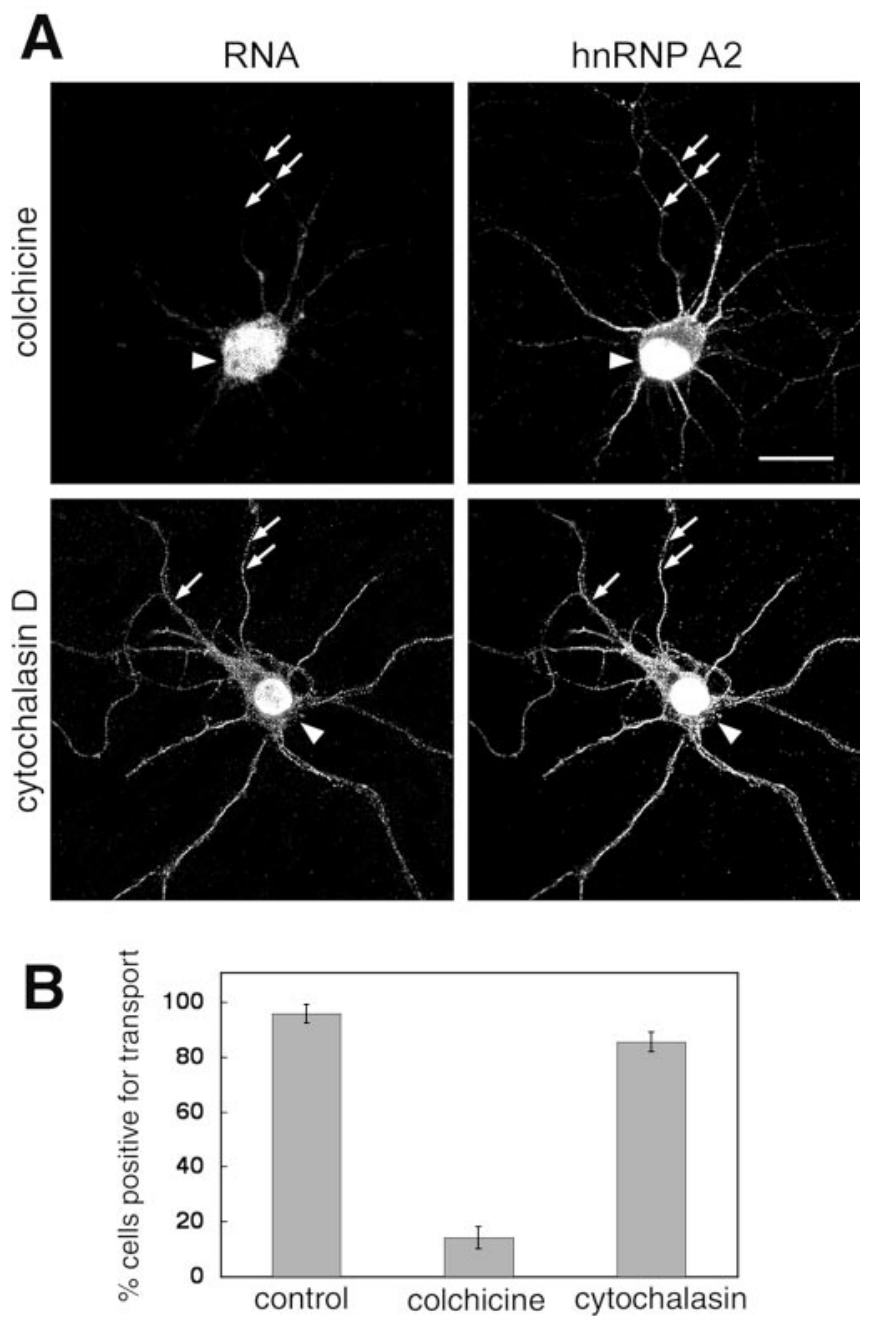

Figure 8. Effects of disruption of microtubules on A2RE RNA trafficking. $A$, The subcellular distribution of microinjected A2RE RNA (left panels) and of endogenous hnRNPA2 (right panels) was analyzed by dual-channel confocal microscopy in cells treated with either colchicine to disrupt microtubules (top panels) or cytochalasin to disrupt microfilaments (bottom panels). hnRNP A2 was detected in granules in the neurites of colchicine-treated cells, but these granules did not contain microinjected RNA (arrows). Scale bar, $15 \mu \mathrm{m}$. B, Percentage of transportpositive cells after treatment with colchicine or cytochalasin. In the control experiment, A2RE RNA was injected into untreated cells. Error bars represent the SDs.

sequence ACCAGGGAGCA, which closely matches the A2RE11, including those nucleotides that are critical for binding to hnRNP A2. In addition, the $\alpha$-CaMKII mRNA contains another A2RElike sequence, C(G,C)CAGAGATCCCTCT, which has been shown to mediate dendritic localization (Mayford et al., 1996). This segment is similar to the $3^{\prime}$ half of A2RE (CAGAGAGCAUG), which binds hnRNP A2 with an affinity comparable with that of the $5^{\prime}$ half of A2RE (i.e., A2RE11) (Munro et al., 1999).

Structural studies of complexes of single-stranded RNAs with RNA recognition motif-containing proteins (such as hnRNP A2) indicate that seven to nine nucleotides are involved in the protein-RNA interface. Consequently, most of the nucleotides in A2RE11 may be required for binding to hnRNP A2. Identification of A2RE-like sequences in other mRNAs depends on the sequence being recognizably similar to the A2RE or A2RE11; however, multiple nucleotide substitutions at some sites in the A2RE11, and more limited changes in others, are tolerated by the transport apparatus (Munro et al., 1999). This sequence flexibility complicates identification of A2RE-like trafficking elements solely on the basis of nucleotide sequence homology. Identification of A2RE-like trafficking elements should be aided by determination of the three-dimensional structure of the hnRNP A2A2RE complex, which we are undertaking.

Other RNA transport and stability elements have been proposed to contain secondary structure motifs, such as stem loops, that are essential for their biological activity. The A2RE and A2RE-like sequences used in our studies are not predicted to have stable secondary structure. Despite the apparent lack of threedimensional structure in the free RNA, the small element size, and the accommodation of nucleotide substitutions at some positions, the A2RE and A2RE11 are recognized specifically by the trafficking apparatus when part of a long RNA molecule.

Are there other cis-acting trafficking or localization elements in neurons?

Trafficking pathways in addition to those involving A2REhnRNP A2 (this work) and zipcode/ZBP1 (Turuchinapalli et al., 2003) may operate in neurons. Each trans-acting factor may also recognize more than one cis-acting element. For example, hnRNP A2 binds both single-stranded DNA and RNA repeats of the telomeric sequence (McKay and Cooke, 1992), which bears no sequence similarity to A2RE, but it does not bind the $\beta$-actin zipcode, the AU-rich element or a number of other oligoribonucleotides (Snee et al., 2002). The binding of RNA to hnRNP A2 is thus sequence selective but not restricted to a single sequence. Furthermore, individual cis-acting elements may bind more than one protein as exemplified by A2RE, which binds both hnRNPs A2 and A3 sequence specifically. Finally, some proteins may have a more general role, influencing the trafficking and localization of multiple RNAs rather than selecting specific mRNAs for transport, as has been proposed for the rat homolog of staufen in dendritic localization (Tang et al., 2001).

In conclusion, the A2RE-hnRNP A2 RNA trafficking pathway functions in neurons and may be responsible for dendritic localization of mRNAs containing A2RE-like elements, including those encoding $\alpha$-CaMKII, neurogranin, and Arc. The evidence linking localization of these three mRNAs to synaptic activity suggests that exploration of the molecular mechanisms for regulation of localization of other mRNAs will be of great interest.

\section{References}

Aakalu G, Smith WB, Nguyen N, Jiang C, Schuman EM (2001) Dynamic visualization of local protein synthesis in hippocampal neurons. Neuron 30:489-502.

Ainger K, Avossa D, Diana AS, Barry C, Barbarese E, Carson JH (1997) Transport and localization elements in myelin basic protein mRNA. J Cell Biol 138:1077-1087.

Barbarese E, Koppel DE, Deutscher MP, Smith CL, Ainger K, Morgan F, Carson JH (1995) Protein translation components are colocalized in granules in oligodendrocytes. J Cell Sci 108:2781-2790.

Bassell GJ, Zhang H, Byrd AL, Femino AM, Singer RH, Taneja KL, Lifshitz LM, Herman IM, Kosik KS (1998) Sorting of $\beta$-actin mRNA and protein to neurites and growth cones in culture. J Neurosci 18:251-265.

Bassell GJ, Oleynikov Y, Singer RH (1999) The travels of mRNAs through all cells large and small. FASEB J 13:447-454.

Blichenberg A, Schwanke B, Rehbein M, Garner CG, Richter D, Kindler S (1999) Identification of a cis-acting dendritic targeting element in MAP2 mRNAs. J Neurosci 19:8818-8829.

Blichenberg A, Rehbein M, Muller R, Garner CG, Richter D, Kindler S (2001) Identification of a cis-acting dendritic targeting element in the mRNA encoding the alpha subunit of $\mathrm{Ca}^{2+} /$ calmodulin-dependent protein $\mathrm{ki}$ nase II. Eur J Neurosci 13:1881-1888.

Bruckenstein DA, Lein PJ, Higgins D, Fremeau Jr RT (1990) Distinct spatial localization of specific mRNAs in cultured sympathetic neurons. Neuron 5:809-819. 
Brumwell C, Antolik C, Carson JH, Barbarese E (2002) Intracellular trafficking of hnRNP A2 in oligodendrocytes. Exp Cell Res 279:310-320.

Carson JH, Worboys K, Ainger K, Barbarese E (1997) Translocation of myelin basic protein mRNA in oligodendrocytes requires microtubules and kinesin. Cell Motil Cytoskeleton 38:318-328.

Carson JH, Kwon S, Barbarese E (1998) RNA trafficking in myelinating cells. Curr Opin Neurobiol 8:607-612.

Chicurel ME, Terrian DM, Potter H (1993) mRNA at the synapse: analysis of a synaptosomal preparation enriched in hippocampal dendritic spines. J Neurosci 13:4054-4063.

Crino PB, Eberwine J (1996) Molecular characterization of the dendritic growth cone: regulated mRNA transport and local protein synthesis. Neuron 17:1173-1187.

Dreyfuss G, Matunis MJ, Piñol-Roma S, Burd CG (1993) hnRNP proteins and the biogenesis of mRNA. Annu Rev Biochem 62:289-321.

Farina KL, Huttelmaier S, Musunuru K, Darnell RB, Singer RH (2003) Two ZBP1 KH domains facilitate $\beta$-actin mRNA localization, granule formation, and cytoskeletal attachment. J Cell Biol 160:77-87.

Frey U, Morris RGM (1997) Synaptic tagging and long-term potentiation. Nature 385:533-536.

Gardiol A, Racca C, Triller A (2001) RNA transport and local protein synthesis in the dendritic compartment. Results Prob Cell Different 34:105-128.

Goslin K, Banker G (1998) Rat hippocampal neurons in low-density culture, Ed 2. Cambridge, MA: MIT.

Hoek KS, Kidd GJ, Carson JH, Smith R (1998) hnRNP A2 selectively binds the cytoplasmic transport sequence of myelin basic protein mRNA. Biochemistry 37:7021-7029.

Kiebler MA, DesGroseillers L (2000) Molecular insights into mRNA transport and local translation in the mammalian nervous system. Neuron 25:19-28.

Kindler S, Monshausen M (2002) Candidate RNA-binding proteins regulating extrasomatic mRNA targeting and translation in mammalian neurons. Mol Neurobiol 25:149-165.

Knowles RB, Sabry JH, Martone ME, Deerinck TJ, Ellisman MH, Bassell GJ, Kosik KS (1996) Translocation of RNA granules in living neurons. J Neurosci 16:7812-7820.

Kohrmann M, Luo M, Kaether C, DesGroseillers L, Dotti CG, Kiebler MA (1999) Microtubule-dependent recruitment of Staufen-green fluorescent protein into large RNA-containing granules and subsequent dendritic transport in living hippocampal neurons. Mol Biol Cell 10:2945-2953.

Kuhl D, Skehel P (1998) Dendritic localization of mRNAs. Curr Opin Neurobiol 8:600-606.

Kwon S, Barbarese E, Carson JH (1999) The cis-acting RNA trafficking signal from myelin basic protein mRNA and its cognate trans-acting ligand hnRNP A2 enhance cap-dependent translation. J Cell Biol 147:247-256.

Landry CF, Watson JB, Kashima T, Campagnoni AT (1994) Cellular influences on RNA sorting in neurons and glia: an in situ hybridization histochemical study. Mol Brain Res 27:1-11.

Lasko P (1999) RNA sorting in Drosophila oocytes and embryos. FASEB J 13:421-433.

Lipshitz HD, Smibert CA (2000) Mechanisms of RNA localization and translational regulation. Curr Opin Genet Dev 10:476-488.

Lisman J, Schulman H, Cline H (2002) The molecular basis of CaMKII function in synaptic and behavioural memory. Nat Rev Neurosci 3:175-190.

Mayford M, Baranes D, Podsypanina K, Kandel ER (1996) The 3'-untranslated region of CaMKII $\alpha$ is a cis-acting signal for the localization and translation of mRNA in dendrites. Proc Natl Acad Sci USA 93:13250-13255.

McKay SJ, Cooke H (1992) hnRNP A2/B1 binds specifically to singlestranded vertebrate telomeric repeat TTAGGGn. Nucleic Acids Res 20:6461-6464.

Miller S, Yasuda M, Coats JK, Jones Y, Martone ME, Mayford M (2002) Disruption of dendritic translation of CaMKIIa impairs stabilization of synaptic plasticity and memory consolidation. Neuron 36:507-519.

Mohr E (1999) Subcellular RNA compartmentalization. Prog Neurobiol 57:507-525.

Mori Y, Imaizumi K, Katayama T, Yoneda T, Tohyama M (2000) Two cisacting elements in the $3^{\prime}$ untranslated region of alpha-CaMKII regulate its dendritic targeting. Nat Neurosci 3:1079-1084.
Mowry KL, Cote CA (1999) RNA sorting in Xenopus oocytes and embryos. FASEB J 13:435-445.

Munro TP, Magee RJ, Kidd GJ, Carson JH, Barbarese L, Smith LM, Smith R (1999) Mutational analysis of a heterogeneous nuclear ribonucleoprotein A2 response element for RNA trafficking. J Biol Chem 274:34389-34395.

Muslimov IA, Santi E, Homel P, Perini S, Higgins D, Tiedge H (1997) RNA transport in dendrites: a cis-acting targeting element is contained within neuronal BC1 RNA. J Neurosci 17:4722-4733.

Nichols RC, Wang XW, Tang J, Hamilton BJ, High FA, Herschman HR, Rigby WFC (2000) The RGG domain in hnRNP A2 affects subcellular localisation. Exp Cell Res 256:522-532.

Oleynikov Y, Singer RH (1998) RNA localization: different zipcodes, same postman? Trends Cell Biol 8:381.

Oleynikov Y, Singer RH (2003) Real-time visualization of ZBP1 association with $\beta$-Actin mRNA during transcription and localization. Curr Biol 13:199-207.

Racca C, Gardiol A, Triller A (1997) Dendritic and postsynaptic localizations of glycine receptor $\alpha$ subunit mRNAs. J Neurosci 17:1691-1700.

Rook MS, Lu M, Kosik KS (2000) CaMKII $\alpha$ 3' untranslated region-directed mRNA translocation in living neurons: visualization by GFP linkage. J Neurosci 20:6385-6393.

Schuman E (1999) mRNA trafficking and local protein synthesis at the synapse. Neuron 23:645-648.

Severt WL, Biber TUL, Wu X-Q, Hecht NB. DeLorenzo RJ, Jakoi ER (1999) The suppression of testis-brain RNA binding protein and kinesin heavy chain disrupts mRNA sorting in dendrites. J Cell Sci 112:3691-3702.

Shyu A-B, Wilkinson MF (2000) The double lives of shuttling mRNA binding proteins. Cell 102:135-138.

Snee MJ, Kidd GJ, Munro TP, Smith R (2002) RNA trafficking and stabilization elements associate with multiple brain proteins. J Cell Sci 115:4661-4669.

Steward O (1997) mRNA localization in neurons: a multipurpose mechanism? Neuron 18:9-12.

Steward O, Halpain S (1999) Lamina-specific synaptic activation causes domain-specific alterations in dendritic immunostaining for MAP2 and CAM kinase II. J Neurosci 19:7834-7845.

Steward O, Levy WB (1982) Preferential localization of polyribosomes under the base of dendritic spines in granule cells of the dentate gyrus. J Neurosci 2:284-291.

Steward O, Worley PF (2001) Selective targeting of newly synthesized Arc mRNA to active synapses requires NMDA receptor activation. Neuron 30:227-240.

Steward O, Wallace CS, Worley PF (1998) Synaptic activation causes the mRNA for the IEG Arc to localize selectively near activated postsynaptic sites on dendrites. Neuron 21:741-751.

Sundell CL, Singer RH (1991) Requirement of microfilaments in sorting of actin messenger RNA. Science 252:1275-1277.

Tang SJ, Meulemans D, Vazquez L, Colaco N, Schuman E (2001) A role for a rat homolog of staufen in the transport of RNA to neuronal dendrites. Neuron 32:463-475.

Tiedge H, Brosius J (1996) Translational machinery in dendrites of hippocampal neurons in culture. J Neurosci 16:7171-7181.

Tiedge H, Chen W, Brosius J (1993) Primary structure, neural-specific expression, and dendritic location of human BC2000 RNA. J Neurosci 13:2382-2390.

Tongiorgi E, Righi M, Cattaneo A (1997) Activity-dependent dendritic targeting of BDNF and TrkB mRNAs in hippocampal neurons. J Neurosci 17:9492-9505.

Torre ER, Steward O (1996) Protein synthesis within dendrites: glycosylation of newly synthesized proteins in dendrites of hippocampal neurons in culture. J Neurosci 16:5967-5978.

Turuchinapalli DM, Oleynikov Y, Kelic S, Shenoy SM, Hartley A, Stanton PK, Singer RH, Bassell GJ (2003) Activity-dependent trafficking and dynamic localization of zipcode binding protein 1 and $\beta$-actin mRNA in dendrites and spines of hippocampal neurons. J Neurosci 23:3251-3261.

Wilhelm JE, Vale RD (1993) RNA on the move: the mRNA localization pathway. J Cell Biol 123:269-274.

Zhang HL, Eom T, Oleynikov Y, Shenoy SM, Liebelt DA, Dictenberg JB, Singer RH, Bassell GJ (2001) Neurotrophin-induced transport of a $\beta$-actin mRNP complex increases $\beta$-actin levels and stimulates growth cone motility. Neuron 31:261-275. 